\title{
The Leica EM VCT100 Versatile Cryo Transfer System.
}

\author{
Kim H. Rensing
}

Leica Mikrosysteme GmbH, Hernalser Hauptstrasse 219, 1170 Vienna, Austria

Bulk samples that are soft or have high water content are very difficult to analyze by electron microscopy, but only this instrumentation offers such high resolution. For roughly 3 decades, chemical preservation (fixation) with plastic embedding was the only real choice to make samples stable for the vacuum and high energy beam in an electron microscope. The drawbacks of this methodology are the slow fixation time (i.e. hours), extraction of sample components, and largescale chemical and structural changes induced by these procedures. However, maturation of cryological techniques over the last 2 decades has given researchers several alternatives for observation of such difficult to work with materials in a more natural condition.

Using a high pressure freezer, samples can be frozen into a vitreous state within milliseconds, often without exposure to chemicals. They can then be dehydrated while in the stable, frozen state via the freeze substitution process, then embedded in resins for sectioning and observation by roomtemperature TEM. This gives a view of biological ultrastructure similar to that of chemical fixation, but greatly improved because it avoids the above-mentioned drawbacks. In addition, it is now possible to observed frozen bulk samples directly by both TEM and SEM. Advances in cryoultramicrotome technology have made cryo-electron microscopy of vitreous sections (CEMOVIS) more easily accessible. The VCT100 versatile cryo transfer system has made it possible to observe both internal and surface structure directly in the frozen state with a high resolution SEM.

The core component of the VCT100 system is a self-contained shuttle which permits movement of frozen samples under vacuum between different instruments that may be some distance apart (Figure 1). Other components convert Leica high-resolution coaters into freeze etching units and convert standard SEMs into cryo-SEMs. With the complete system, material can be coated while frozen to observe outer surfaces or freeze fractured and coated to observe inner structure. Samples can be then moved without contamination into the cryo-SEM and observed with high resolution (Figure 2). The entire process from freezing through to imaging can be performed in less than 30 minutes, making this suitable for high throughput situations. This technology is thus useful for a variety of materials ranging from biological samples to industrial materials. 


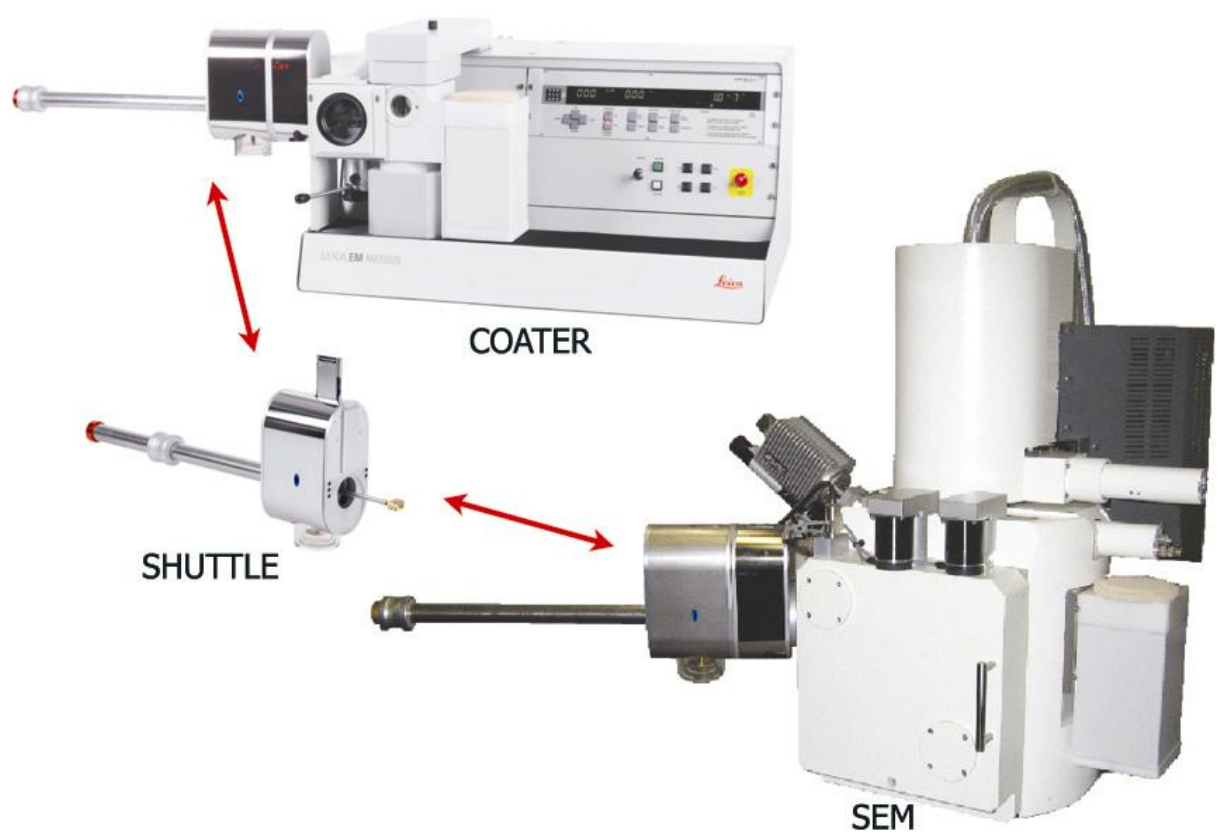

Figure 1. The VCT100 shuttle transfers frozen samples between a fracturing/coating system and a cryo-SEM.

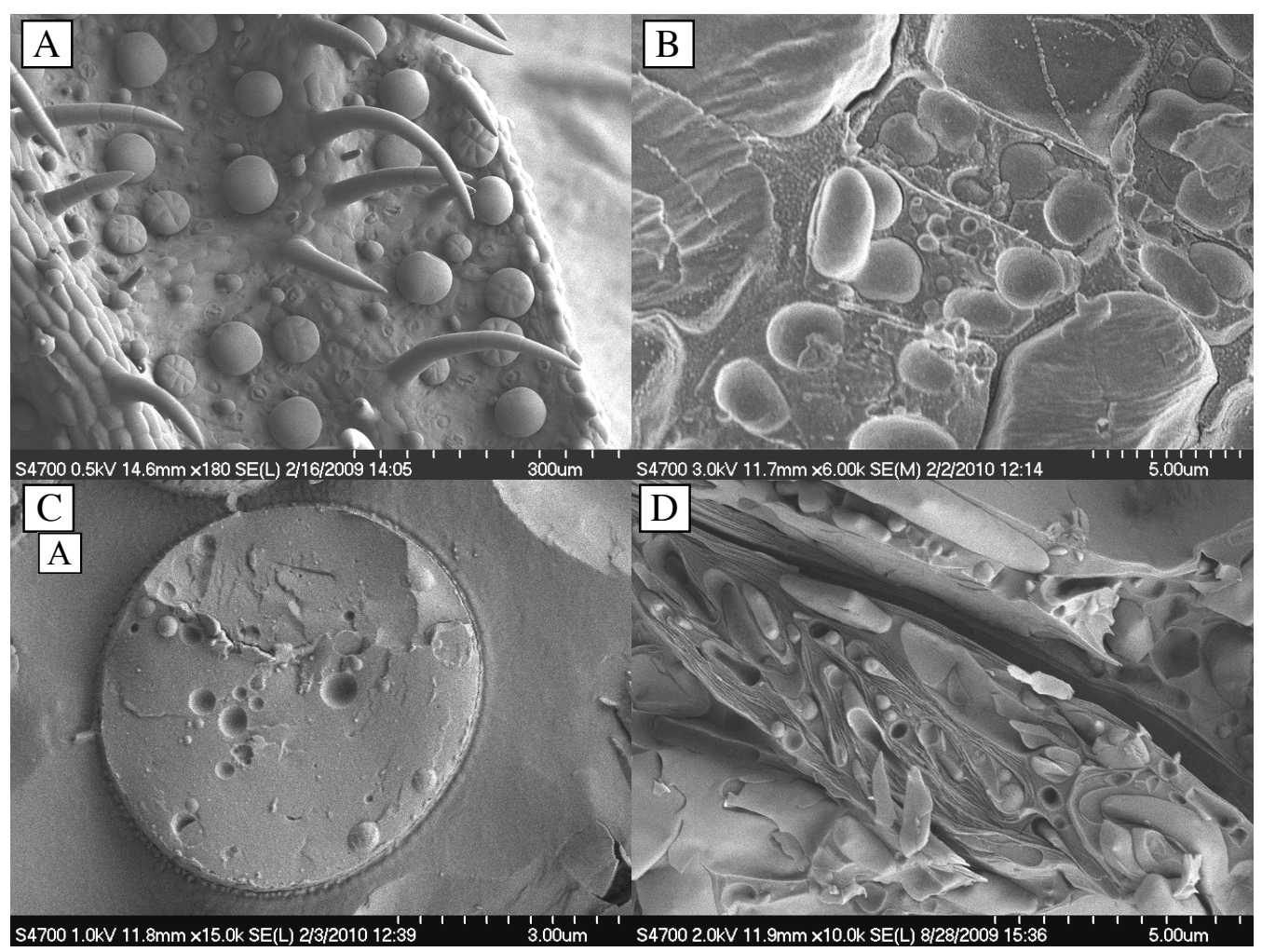

Figure 2. CryoSEM and VCT100 results of different samples. A. Mint leaf surface showing the distribution of two kinds of trichomes. B. Freeze fractured Arabidopsis stem epidermal layer. C. Freeze fractured yeast. D. Freeze fractured skin moisturizer shows the structure of an aqueous emulsion. 